Utah State University

DigitalCommons@USU

2013

\title{
Electron Transport Models and Precision Measurements with the Constant Voltage Conductivity Method
}

Justin Dekany

JR Dennison

Utah State Univesity

Alec Sim

Irvine Valley Collge

Jerilyn Brunson

Naval Surface Warfare Center, Dahlgren Division

Follow this and additional works at: https://digitalcommons.usu.edu/graduate_pubs

Part of the Physics Commons

\section{Recommended Citation}

Justin Dekany, Alec M. Sim, Jerilyn Brunson, and JR Dennison, "Electron Transport Models and Precision Measurements with the Constant Voltage Conductivity Method," IEEE Trans. on Plasma Sci., 41(12), 2013, 3565-3576. DOI: 10.1109/TPS.2013.2288366

This Article is brought to you for free and open access by the Browse all Graduate Research at DigitalCommons@USU. It has been accepted for inclusion in Graduate Student Publications by an authorized administrator of DigitalCommons@USU. For more information, please contact

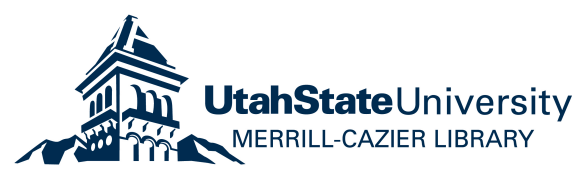




\title{
Electron Transport Models and Precision Measurements with the Constant Voltage Conductivity Method
}

\author{
Justin Dekany, JR Dennison, Alec M. Sim and Jerilyn Brunson
}

\begin{abstract}
Recent advances are described in the techniques, resolution, and sensitivity of the Constant Voltage Conductivity (CVC) method and the understanding of the role of charge injection mechanisms and the evolution of internal charge distributions in associated charge transport theories. These warrant reconsideration of the appropriate range of applicability of this test method to spacecraft charging. We conclude that under many (but not all) common spacecraft charging scenarios, careful CVC tests provide appropriate evaluation of conductivities down to $\approx 10^{-22}(\Omega-\mathrm{cm})^{-1}$, corresponding to decay times of many years.

We describe substantial upgrades to an existing CVC chamber, which improved the precision of conductivity measurements by more than an order of magnitude. At room temperature and above and at higher applied voltages, the ultimate instrument conductivity resolution can increase to $\approx 4 \cdot 10^{-22}(\Omega-\mathrm{cm})^{-1}$, corresponding to decay times of more than a decade. Measurements of the transient conductivity of low density polyethylene (LDPE) using the CVC method are fit very well by a dynamic model for the conductivity in highly disordered insulating materials over more than eight orders of magnitude in current and more than six orders of magnitude in time. Current resolution of the CVC system approaches fundamental limits in the laboratory environment set by the Johnson thermal noise of the sample resistance and the radiation induced conductivity from the natural terrestrial background radiation dose from the cosmic ray background.
\end{abstract}

Index Terms-Conductivity, insulator, dielectric materials, electron transport, charge storage, instrumentation

\section{INTRODUCTION}

Tnvestigations of the complex interplay between dielectric 1 spacecraft components and their charging space plasma

Research was supported by funding from the NASA James Webb Space Telescope Program through Goddard Space Flight Center, the NASA Space Environments and Effects Program, NASA Rocky Mountain Space Grant Consortium graduate fellowships for Sim and Brunson, and Utah State University Undergraduate Research and Creative Opportunities grants for Brunson and Dekany. Dennison acknowledges support from the Air Force Research Laboratory through a National Research Council Senior Research Fellowship. Dekany and Dennison are with the Materials Physics Group in the Physics Department at Utah State University in Logan, UT 84322 USA (e-mail: JDekany.phyx@gmail.com, JR.Dennison@usu.edu). Brunson is with the Naval Surface Warfare Center Dahlgren Division in Dahlgren, VA 22448 (email: Jerilyn.Brunson@navy.mil). Sim is with Irvine Valley College, Irvine CA 92618 USA (e-mail: ASim@ivc.edu ).

Color versions of one or more figures in this paper are available online at http://ieeexplore.ieee.org.

Digital object identifier . environments are fundamentally based on a detailed knowledge of how individual materials store and transport charge. The low charge mobility of insulators causes charge to accumulate where deposited, preventing uniform redistribution of charge and creating differential local electric fields and potentials. The conductivity of spacecraft materials is the key transport parameter in determining how deposited charge will redistribute throughout the system, how rapidly charge imbalances will dissipate, what equilibrium potential will be established under given environmental conditions, and ultimately if and when electrostatic discharge will occur [1-3].

Comparison of characteristic charge accumulation times for spacecraft (e.g., rotational periods, orbital periods, mission lifetimes, or times for materials modifications such as accumulation of contaminates or evolution due to environmental fluxes) to charge dissipation times (e.g., the transit time or charge decay time $\tau=\varepsilon_{o} \varepsilon_{r} / \sigma$, where $\varepsilon_{o}$ is the permittivity of free space and $\varepsilon_{r}$ is the relative permittivity) have been used to establish ranges of conductivity, $\sigma$, that are to be viewed with concern for spacecraft charging [4-6]. For example, if the charge decay time exceeds the orbital period, not all charge will be dissipated before orbital conditions act again to further charge the satellite. As the insulator accumulates charge, the electric field will rise until the insulator breaks down. Thus, charge decay times in excess of $\sim 1 \mathrm{hr}$ are problematic, as is specifically stated in NASA Handbook 4002 [4]. Considering these results [6], marginally dangerous conditions begin to occur for materials with conductivities less than $\sim 10^{-16}(\Omega-\mathrm{cm})^{-1}$ with $2<\varepsilon_{\mathrm{r}}<4$, when $\tau$ exceeds $\sim 1 \mathrm{hr}$. More severe charging conditions occur for $\left(\varepsilon_{o} \varepsilon_{r} / \tau\right) \leqslant 10^{-18}(\Omega-\mathrm{cm})^{-1}$, when decay times exceed $\sim 1$ day. Extreme insulators with decay times in excess of mission lifetimes (e.g., $\tau>2$ decades or $\sigma \lesssim 4 \cdot 10^{-22}(\Omega \text {-cm })^{-1}$ ) can effectively be treated as "perfect charge integrators". Thus, measurements of conductivities beyond this extreme are not necessary for spacecraft charging predictions.

Existing spacecraft charging guidelines [4,5] recommend use of standard conductivity tests and imported conductivity data from handbooks that are based principally upon ASTM methods [7]. These methods are more applicable to classical ground conditions and designed for problems associated with power loss through the dielectric, than for how long charge can be stored on an isolated insulator. These data have been found to underestimate charging effects by one to four orders of magnitude for many spacecraft charging applications $[8,9]$. 
Based on these time comparisons and related issues, and on the ranges of conduction that could be measured with different methods, Frederickson [10,11] and Swaminathan [12,13] have made recommendations for amendments to NASA Handbook 4002 [4] as to preferred methods and improvements to determine conductivity of dielectric spacecraft materials. Two higher precision test methods identified in ASTM D-257 were recommended for low conductivity measurements for spacecraft charging applications, the Constant Voltage Conductivity (CVC) and Charge Storage Conductivity (CSC) methods. They recommended that these higher precision tests must be conducted in stringent test conditions under vacuum with apparatus that are well designed to minimize problems from sample contamination, temperature, humidity, vibration, electromagnetic interference, dielectric breakdown and other confounding variables as outlined in ASTM D-257 [7] and ASTM 618 [14]. Contrary to ASTM D-257 guidelines that suggest a measurement settling time of only 1 min [7], the higher precession tests of spacecraft insulators must be conducted over long enough durations to assure that the material conductivity has come to equilibrium; this may require from minutes to months depending on the materials being tested $[6,12,13]$. Based primarily on the minimum measurable conductivities for the two methods, Swaminathan $[12,13]$ concluded that such a CVC method is usually most appropriate for materials with conductivities in a range of $10^{-13}$ $(\Omega-\mathrm{cm})^{-1}>\sigma>10^{-17}(\Omega-\mathrm{cm})^{-1}$ (or equivalently $1 \mathrm{sec}>\tau>10 \mathrm{hr}$ ), while the CSC method is the method of choice for very low conductivity materials with $\sigma<10^{-16} \Omega$-cm or $\tau>1 \mathrm{hr}$.

Recent advances have been made in the techniques, resolution, and sensitivity of both the CVC [15-17] and CSC [18-22] methods and also in the understanding of the associated charge transport theories [21-23]. These improvements warrant revisiting the discussion of the appropriate range of applicability to spacecraft charging of these two test methods.

We begin this paper with a review of improvements in instrumentation and measurement methods that have significantly extended the range of the CVC method. This is accompanied by a review of the advances of our theoretical understanding of the role of charge injection mechanisms and the evolution of internal charge distributions, and how these differ for the CVC and CSC methods. Measurements and theoretical limits for the detection threshold for CVC methods are then presented. We end with a discussion of the best choice of conductivity test methods for ranges of conductivity values and space environment scenarios. We also comment on which test method best models different charging conditions encountered in space applications.

\section{CVC INSTRUMENTATION}

Figure 1 illustrates the basic configurations for the CVC and CSC methods. The CVC method (see Fig. 1(a)) applies a constant voltage to a front electrode attached to the sample in a parallel plate configuration, resulting in an injection current density, $J_{i n j}(t)$, into the sample $[13,16,17,22,24]$. The current at a grounded rear electrode is measured as a function of time. The conductivity of a material is determined by

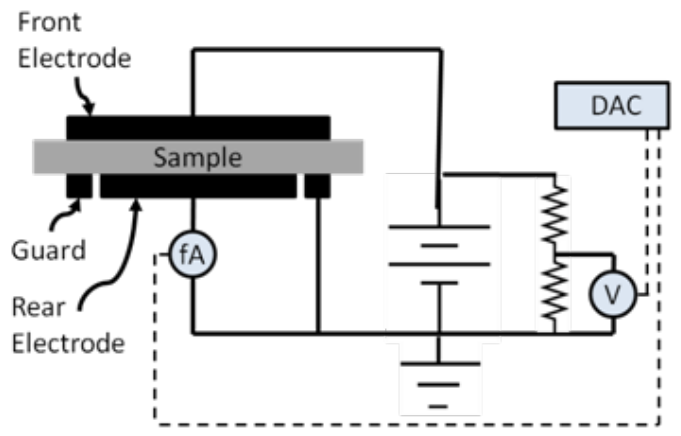

(a)

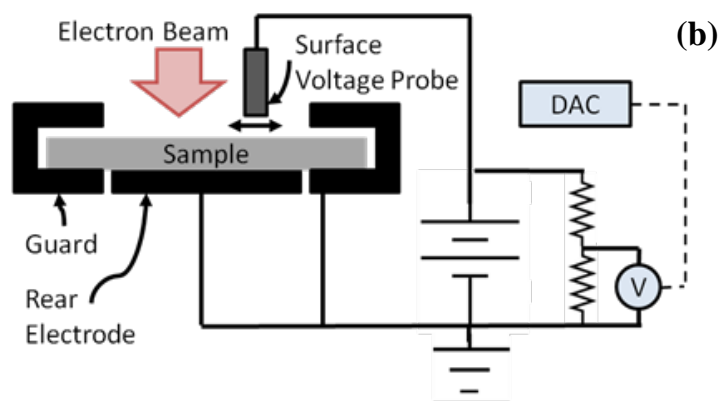

Fig. 1. Simplified schematics of (a) Constant Voltage Conductivity (CVC) and (b) Charge Storage Conductivity (CSC) test circuits.

$$
\sigma(t)=\frac{J_{\text {elec }}(t)}{F(t)}=\frac{I_{\text {elec }}(t) D}{A V_{a p p}(t)}
$$

based on four measured quantities: sample area, $A$; sample thickness, $D$; rear electrode current, $I_{\text {elec }}$; and applied voltage, $V_{\text {app }}$.

By contrast, the CSC method (see Fig. 1(b)) monitors the front surface voltage of the sample as a function of time, using a noncontact electric field probe $[3,8-10,13,15,18-22]$. The voltage, measured with respect to the grounded rear electrode, results from the internal charge distribution within the sample, most often embedded in the sample with electron beam injection over a short time span at the start of a measurement.

\section{A. Description of the USU CVC System}

The instrumentation used at Utah State University (USU) to measure conductivity of highly resistive dielectric materials using the CVC method is described below, with particular attention given to the lower threshold of conductivity that it can measure. The chamber (see Fig. 2) provides a highly stable controlled vacuum, temperature, and noise environment for long-duration conductivity measurements over a wide range of temperatures ( $<100 \mathrm{~K}$ to $\sim 350 \mathrm{~K}$ ) and electric fields (up to the breakdown voltages for many common materials). A new system, with similar design, has recently been developed that uses a closed-cycle He cryostat to extend measurements down to $<40 \mathrm{~K}$ [25].

The CVC system has undergone numerous revisions, in both its electronic and hardware configurations, to reduce the measured conductivity threshold. This includes extensive refinements of shielding, ground loop, computer interfacing, and noise issues that have substantially lowered the baseline of electrical noise. Resolving these issues has improved the accuracy and precision of current measurements to as low as $2 \cdot 10^{-16} \mathrm{~A}[16]$. Modifications to the applied voltage sources, 
the current and voltage monitoring circuits, the data acquisition system, and the data analysis are described in Appendix A, which emphasizes the uncertainties in the CVC measurements. Further details of the original instrumentation [15] and of the CVC chamber enhancements [6,16] are found elsewhere.

\section{B. Uncertainties for the CVC System}

The magnitudes of systematic and random errors and their relative contribution to the total error in conductivity are addressed below; further details are provided in Appendix A and [6].

The accuracy of the conductivity measurements is driven by absolute uncertainties in sample area and thickness, except for the very lowest conductivities where uncertainties due to current measurements and voltage fluctuations dominate. The $(1.59 \pm 0.03) \mathrm{cm}$ diameter oxygen-free, high-conductivity (OFHC) $\mathrm{Cu}$ electrodes have an effective contact area of $(1.98 \pm 0.08) \mathrm{cm}^{2}$ (as corrected for fringe fields, guard rings and electrode geometry [7]), with an accuracy of $\pm 4 \%$ [6]. Variations in the contact area of the electrode have been reduced by the addition of a sample clamping fixture (see Fig. 2(b)). Typical sample thicknesses of $10 \mu \mathrm{m}$ to $200 \mu \mathrm{m}$ were measured with a resolution of $\pm 0.3 \mu \mathrm{m}$. Systematic uncertainties from variations in the thicknesses of typical samples were comparable to this precision. Since both effective sample area and mean thickness are fixed for a given CVC measurement, their uncertainties affect the accuracy of conductivity measurements, but not the precision.

The relative random error in conductivity is obtained by addition in quadrature of the relative random errors of the four measured quantities in (1):

$\frac{\Delta \sigma}{|\sigma|}=\left[\left(\frac{\Delta A}{|A|}\right)^{2}+\left(\frac{\Delta D}{|D|}\right)^{2}+\left(\frac{\Delta I_{\text {elec }}}{\left|I_{\text {elec }}\right|}\right)^{2}+\left(\frac{\Delta V_{\text {app }}}{\left|V_{\text {app }}\right|}\right)^{2}\right]^{1 / 2}$.

At short times conductivity resolution is on the order of a few percent, set primarily by the changes in conductivity over the sampling times to acquire current and voltage measurements and by the uncertainties in area and sample thickness. At long times, conductivity resolution is limited by absolute instrumental resolution of current measurements and by noise in the current measurements due to fluctuations in applied voltage.

The estimated precision for mean current measurements, $(\Delta I /|I|)$, over a range of $10^{-6} \mathrm{~A}$ to $10^{-16} \mathrm{~A}$ is $\lesssim 0.1 \%$ at $>1 \cdot 10^{-11}$ A and $\gtrsim 20 \%$ at $\lesssim 1 \cdot 10^{-15} \mathrm{~A}$. At typical measured currents, the contributions to uncertainties due to the electrometer dominate current measurements. The electrometer instrument error values of $\sim 2 \cdot 10^{-16} A$ represents the lowest possible current measurement that can be taken with our present system, which is on the order of $~ 250$ electrons per current measurement. Residuals from fits to our models for data presented in Section V.B of $\sim 2 \cdot 10^{-18} \mathrm{~A}$ (or $\sim 12$ electrons/s) are equivalent to \pm 1 electron per measurement sampled at $10 \mathrm{~Hz}$.

Uncertainties due to voltage sources enter in several ways. Variation in accuracy of the applied voltage (due primarily to long-term drift of the voltage supply), are directly monitored with the data acquisition card (DAC) and are compensated for
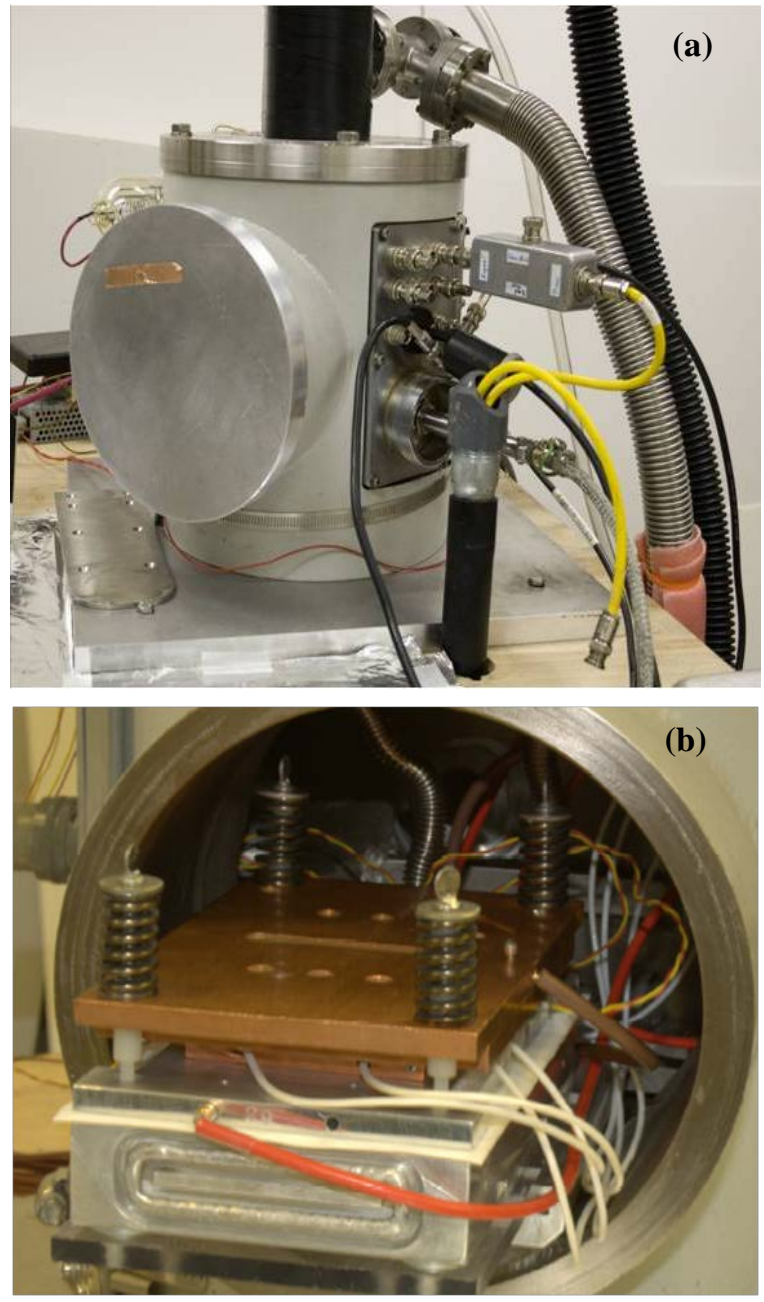

Fig. 2. Constant voltage conductivity (CVC) chamber. (a) Exterior view. Shown are sample access port (lower left), vacuum electrical feedthroughs attached signal triaxial cable with vibrational stabilization (lower right), vacuum pumping port, and liquid nitrogen port (top). (b) Interior view. CVC experimental plate stack shown with the thermal radiation shield removed. Aluminum temperature reservoir (bottom) is isolated from the Al voltage half-plates by a thin layer of Chotherm ${ }^{\mathrm{TM}}$. Four spring clamps at each corner maintain constant pressure on samples.

in the conductivity calculations using (1). Random uncertainties in $V_{a p p}$ enter directly through the last term of (2). These relative errors range from $\sim 0.7 \%$ to $\sim 0.1 \%$ for two different programmable DC voltage sources used with our CVC system (see Appendix A). At voltages below $400 \mathrm{~V}$, the instrumental precision of voltage measurements depends primarily on the DAC, while above this voltage errors from the voltage supply increase to about twice the DAC error. For measured currents $\gtrsim 1 \cdot 10^{-11} \mathrm{~A}$ this is the dominate term for (2).

More importantly, small short time scale fluctuations in $V_{a p p}$ lead to uncertainties in $I_{\text {elec }}(t)$ through the displacement and polarization terms of (4). These terms can be significant as $\sigma(t) \rightarrow \sigma_{\text {sat }}$, even for small changes in $V_{\text {app}}$, since the polarization and displacement currents are much larger than the saturation current at times immediately following a change in injected charge due to a fluctuation in applied voltage.

To minimize these contributions to $\Delta \sigma /|\sigma|$ from $V_{\text {app }}$, a very low-noise low-voltage $100 \mathrm{~V}$ battery source was constructed with $\Delta V_{o} \approx 16 \mathrm{mV}$ and $\Delta V_{\text {rel }} /|V| \approx 0.02 \%$. Uncertainties result largely from the voltage monitoring 
The effectiveness of all of these efforts to minimize uncertainties is addressed in Section IV.A.

\section{CONDUCTIVITY THEORY}

To understand the subtle differences in CVC and CSC measurements a detailed theoretical description of the various contributions to the time-dependant conductivity and rear electrode current are developed in Appendix B. For the CVC and CSC experimental conditions considered here, the generalized time-dependant non-Ohmic conductivity for highly disordered insulating materials (HDIM) [26] given by (B1) is restricted so that:

(i) AC conductivity is excluded for non-periodic voltages;

(ii) RIC is excluded for CVC electrode injection; and

(iii) saturation current is excluded for CSC pulsed injection. From (B1), this leaves an expression for the CVC conductivity of

$\begin{aligned} \sigma_{\mathrm{CVC}}(\mathrm{t})=\sigma_{\mathrm{Sat}}+\sigma_{\mathrm{pol}}^{o} \mathrm{e}^{-\mathrm{t} / \tau_{\mathrm{pol}}} & +\left[\sigma_{\text {diffusion }}^{o} t^{-1}+\sigma_{\text {dispersive }}^{o} t^{-(1-\alpha)} \Theta\left(\tau_{\text {transit }}-t\right)\right. \\ & \left.+\sigma_{\text {transit }}^{o} t^{-(1+\alpha)} \Theta\left(t-\tau_{\text {transit }}\right)\right]\end{aligned}$

and for the CSC conductivity of

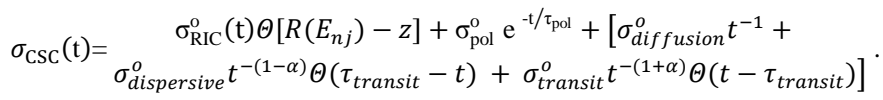

Combining an expression for the free electron charge transport current density based on the results of (3) with explicit expressions for the polarization current from (B3) and the displacement current from (B7), we have an explicit expression for the rear electrode current,

$$
\begin{array}{r}
J_{\text {elec }}(t)=F_{\text {app }}\left\{\left[\sigma(\mathrm{t})+\sigma_{\text {pol }}^{o} \cdot e^{-t / \tau_{p o l}}\right]\left(1-e^{-t / \tau_{Q}}\right)-\right. \\
\left.\left(\frac{\varepsilon_{o} \varepsilon_{r}}{\tau_{Q}}\right) e^{-t / \tau_{Q}}\right\},
\end{array}
$$

where $\sigma(t)$ is given by the more general expression (B2) or (3a) or (3b) for the CVC or CSC systems, respectively. [Note, for clarity, the polarization contribution is shown explicitly in (4), even though it has been included in (B2) and (3).]

In most cases, the displacement current from (4) and those from transient currents dominate on different time scales, and can hence be easily separated in the analysis (as we do in Section V.B). At short times, the first term in (4), $\sigma(t) F_{a p p}(t)$ is small and the polarization current and the displacement current from (B3) and (B7) dominate, giving

$J_{\text {elec }}^{\text {small } t}(t)=F_{\text {app }}\left[\sigma_{\text {pol }}^{o} \cdot e^{-t / \tau_{\text {pol }}}\left(1-e^{-t / \tau_{Q}}\right)-\left(\frac{\varepsilon_{o} \varepsilon_{r}}{\tau_{Q}}\right) e^{-t / \tau_{Q}}\right]$.

After a relatively short period of time $F_{a p p}(t)$ and the polarization become constant, the currents in (5) become negligible, and the terms associated with $\sigma(t) F_{a p p}(t)$ (including the transient currents) dominate in (4).

\section{DETERMination Of DETECTION THRESHOLD}

To address the question of the range of applicability of the improved CVC method, we compare the measured detection threshold and noise levels, a detailed error analysis of the system, and some fundamental limits to current detection with the CVC method.

\section{A. Measured Noise Threshold}

By comparing the statistical error in measured current data to the instrument error for three data sets shown in Fig. 3, we can assess the enhancements to the CVC chamber described above and determine a quantitative measure of the lowest conductivity measurable with the instrument in each stage of the upgrades. Figure 3(a) shows data taken prior to the modifications to the CVC chamber described in Section II and Appendix A; the statistical errors of conductivity for this data set are relatively large (green lines, spanning almost an order of magnitude). Figure 3(b) shows data taken after the spring clamping system was installed and vacuum issues were corrected; the adaptive smoothing algorithm was also applied to these data. The instrumentation (red curves) and statistical errors (green curves) were greatly reduced. Figure 3(c) shows data taken with the improvements used in Fig. 3(b), plus the use of a $100 \mathrm{~V}$ highly stable battery voltage supply. Note that the \pm 1 standard deviation statistical error limits (green lines in Fig. 3(c)) for this data set have been reduced even further and are approaching the theoretical limit of the instrument errors for current (red lines in Fig. 3).

The average long-term equilibrium dark current conductivity value of $\sim 9 \cdot 10^{-19}(\Omega \text {-cm })^{-1}$ for low-density polyethylene (LDPE) samples obtained in all three tests the CVC agrees with literature for measurements taken at room temperature [24]. Average (smoothed) conductivity values (blue lines) for Figs. 3(b) and 3(c) obtained after the chamber modifications agree to within $\sim 10 \%$; they also are within $\sim 50 \%$ of the values in Fig. 3(a) obtained with data taken prior to the modifications. The statistical error in current shows a reduction of greater than $\sim 50 \%$ from Fig. 3(a) to Fig. 3(b) and a reduction of $\sim 90 \%$ from data in Fig. 3(a) to data in Fig. 3(c); this equates to roughly an order of magnitude increase in the precision of current measurements obtained with the CVC.

The conductivity instrument error of $3 \cdot 10^{-21}(\Omega-\mathrm{cm})^{-1}$ for data in Figs. 3(a-c) at the lowest sensitivity setting represents the lowest threshold limit for conductivity measurements made using the CVC chamber in its present modified configuration; this has a corresponding longest measurable decay time of $\geq 2.5$ yr. Planned implementation of an equally stable $1000 \mathrm{~V}$ higher voltage battery voltage supply [17] will allow a $10 \mathrm{X}$ increase in longest measurable decay time and a corresponding $\sim 10 \mathrm{X}$ decrease in effective $\Delta I /|I|$. Assuming that $\Delta \sigma / \sigma$ is dominated by the $\Delta I /|I|$ term when using the highly stable battery supply, the mean precision for time decay will decrease to $\sim 4 \cdot 10^{-22}(\Omega-\mathrm{cm})^{-1}$ with a corresponding decay time of $\geq 20$ yr. The estimated ultimate instrument conductivity resolution is $\sim 4 \cdot 10^{-23}(\Omega-\mathrm{cm})^{-1}$ or a decay time of $>2$ centuries, for a upper bound of the applied voltage of 8200 $\mathrm{V}$ approaching the breakdown voltage for a $27 \mu \mathrm{m}$ thick LDPE sample. This ultimate resolution of the CVC chamber can be compared to fundamental limits inherent in the environment. 


\section{B. Johnson Current Limit}

A fundamental limit to measurement of current or conductivity is the Johnson noise of the source resistance. For any resistance, thermal energy produces motion of the constituent charged particles, which results in what is termed Johnson or thermal noise. The peak to peak Johnson current noise of a resistance $\mathfrak{R}$ at temperature $\mathrm{T}$ is [27]:

$$
\Delta I_{J N}=5 \sqrt{\frac{4 k_{B} T W_{\text {Band }}}{\mathfrak{R}}},
$$

where $W_{\text {Band }}$ is the signal band width approximated as $\left(0.35 / T_{\text {Rise }}\right)$ [27]; for the lowest $10^{-11}$ A electrometer range, this is $\sim 3 \mathrm{~s}$ and $T_{\text {Rise }} \approx 0.1 \mathrm{~Hz}$ [28]. For a typical LDPE sample at room temperature $\Delta I_{J N} \approx 4 \cdot 10^{-18}$ A with a corresponding $\sigma_{J N} \approx 6 \cdot 10^{-23}(\Omega-\mathrm{cm})^{-1}$ at $100 \mathrm{~V}$; this is $\sim 2 \%$ of the ultimate instrument conductivity resolution at $100 \mathrm{~V}$. For a typical LDPE sample at $\sim 100 \mathrm{~K}, \Delta I_{J N} \approx 3 \cdot 10^{-19}$ A with a corresponding $\sigma_{\mathrm{JN}} \approx 5 \cdot 10^{-24}(\Omega-\mathrm{cm})^{-1}$ at $100 \mathrm{~V}, \sim 0.2 \%$ of the ultimate instrument conductivity resolution at $100 \mathrm{~V}$ calculated above. At an upper bound of $8200 \mathrm{~V}$, the Johnson current noise at room temperature is $\sim 200 \%$ of the ultimate instrument conductivity resolution calculated above, and $\sim 15 \%$ at $100 \mathrm{~K}$.

\section{Background Radiation Limit}

Another limit to the conductivity results from interaction with the natural background radiation environment. The worldwide average natural background radiation dose from the cosmic ray background at sea level is $\sim 0.26 \mathrm{mGy} / \mathrm{yr}$ [29]. This is increased by a factor of about $75 \%$ at an altitude of $1400 \mathrm{~m}$ in Logan, UT [29]. Radiation from other sources of background radiation including terrestrial sources such as soil and radon gas, as well as man-made sources, are typically not high enough energy to penetrate the CVC vacuum chamber walls, and are hence shielded and not considered in this calculation. By contrast, cosmic ray background radiation is of high enough energy to have penetrated the atmosphere and so will not be appreciably attenuated by building or chamber walls. Our calculation also does not take in to account any charge deposited by the cosmic ray radiation or secondary charge emitted by the sample or electrodes in contact with the sample; these could conceivably be significant factors.

Our natural cosmic background annual dose is $\sim 0.46 \mathrm{mGy}$, with an average dose rate of $1.4 \cdot 10^{-11} \mathrm{~Gy} / \mathrm{s}$. Using values of $k_{R I C}=2 \cdot 10^{-14}(\Omega-\mathrm{cm}-\mathrm{Gy} / \mathrm{s})^{-1}$ and $\Delta=0.8$ for LDPE at room temperature [30], this corresponds to a background $\sigma_{R I C}$ of $\sim 4 \cdot 10^{-23}(\Omega-\mathrm{cm})^{-1}$. This is $\sim 1 \%$ of the ultimate instrument conductivity resolution at $100 \mathrm{~V}$ applied voltage or about equal to the ultimate instrument conductivity resolution for our upper bound of $8200 \mathrm{~V}$.

\section{Comparison of Detection Limits}

Thus, in summary, the fundamental limit of the CVC system is set:

- at low temperatures, by the ultimate instrument conductivity resolution;

- $\quad$ at room temperature and lower voltages, by the ultimate instrument conductivity resolution; and

- $\quad$ at room temperature and highest voltages, by nearly equal contributions (in decreasing order) from the ultimate

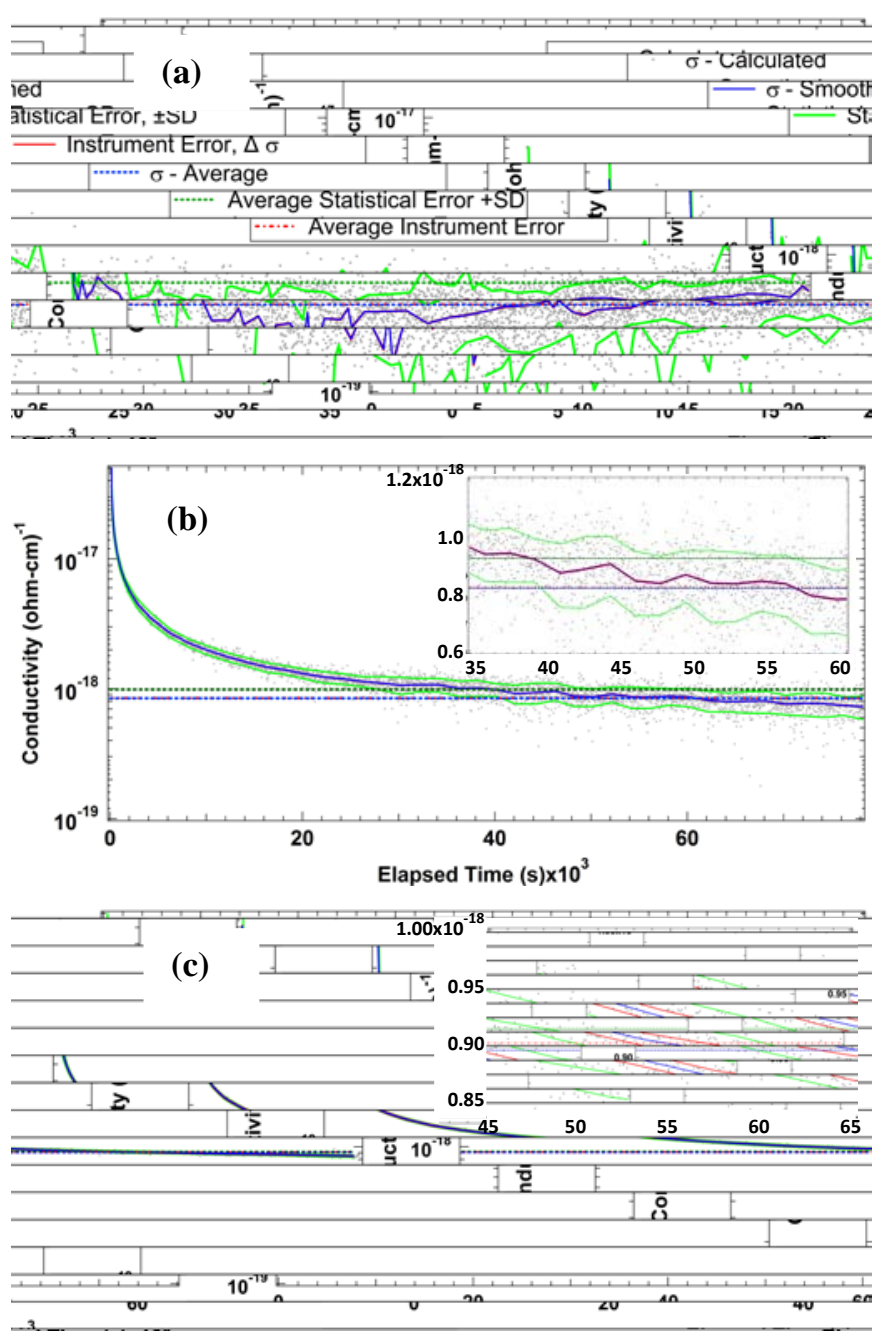

Fig. 3. Comparison of precision of conductivity versus time data runs for sequential improvements in CVC instrumentation: (a) Conductivity data prior to chamber modifications using a filtered medium voltage source; (b) Conductivity data after chamber modifications and applying CVC analysis algorithm using a filtered medium voltage source; and (c) conductivity data after chamber modifications and applying the CVC analysis algorithm, using an isolated battery power supply. Data were acquired for a constant $\sim 100 \mathrm{~V}$ nominal voltage for $\sim 96 \mathrm{hr}$ at variable temperature with a $27.4 \mu \mathrm{m}$ thick LDPE sample. Data sets acquired at $20 \mathrm{~s}$ intervals are shown as grey dots. Smoothed values from a dynamic binning and averaging algorithm are shown in blue. Green lines show statistical errors for the binned and averaged data at \pm 1 standard deviation. The red curves show the estimated instrumental uncertainty based on (2). The insets show linear plots of the data and errors near the equilibrium current.

instrument conductivity resolution, thermal noise, and equilibrium $\sigma_{R I C}$ from cosmic ray background radiation.

At short times and higher currents, precision of conductivity measurements is limited to a few percent, set primarily by the changes in conductivity over the times to measure the current and voltage and the uncertainties from voltage supplies. At long times and lower currents using highly stable voltage supplies, conductivity resolution is limited by absolute instrumental current resolution (which approaches fundamental limits set by the thermal Johnson noise and background radiation).

For our existing system, using a $100 \mathrm{~V}$ battery voltage source, the instrument conductivity resolution of $\sim 4 \cdot 10^{-21}(\Omega$ $\mathrm{cm})^{-1}$ (equivalent to $\tau \lesssim 3 \mathrm{yrs}$ ) is less than the lower bound of conductivities relevant to spacecraft applications of $\gtrsim 4 \cdot 10^{-22}$ 
$(\Omega \text {-cm) })^{-1}$ (equivalent to mission lifetimes of $\tau<2$ decades). This limit can easily be reached with the use of higher $\mathrm{kV}$ voltage battery sources.

\section{ANALYSIS OF CVC RESULtS}

\section{A. CVC Sample Characteristics}

Samples of branched, low-density polyethylene (LDPE) of (27.4 \pm 0.2$) \mu \mathrm{m}$ thickness had a density of $0.92 \mathrm{~g} / \mathrm{cm}^{3}$ [31] with an estimated crystallinity of 50\% [32] and a relative dielectric constant of 2.26 [31]. All samples were chemically cleaned with methanol prior to a bakeout at $65( \pm 1){ }^{\circ} \mathrm{C}$ under $\sim 10^{-3} \mathrm{~Pa}$ vacuum for $>24 \mathrm{hr}$ to eliminate absorbed water and volatile contaminants; samples conditioned in this manner had a measured outgassing rate of $<0.05 \%$ mass loss/day at the end of bakeout, as determined with a modified ASTM 495 test procedure [33]. Electrostatic breakdown field strength of conditioned samples was measured in a separate test chamber to be $(2.9 \pm 0.3) \cdot 10^{8} \mathrm{~V} / \mathrm{m}$, using a modified ASTM D 3755 test procedure [34] at room temperature under $<10^{-2} \mathrm{~Pa}$ vacuum with a voltage ramp rate of $20 \mathrm{~V}$ steps each 4 second. A similar test, conducted in the CVC chamber at a voltage ramp rate of $50 \mathrm{~V}$ steps each second, found an electrostatic breakdown field strength of $2.6 \cdot 10^{8} \mathrm{~V} / \mathrm{m}$.

\section{B. Fits to CVC Data}

To illustrate some of the capabilities of the CVC chamber, we provide a qualitative assessment of measurements of the rear electrode current. The representative data and associated fits for LDPE shown in Fig. 3 span more than eight orders of magnitude in current and six orders of magnitude in time. At long times, typical residuals for the fit to smoothed data are in the range of $10^{-18} \mathrm{~A} / \mathrm{cm}^{2}$.

The initial time-dependence of the rear electrode current in the first $4 \mathrm{~s}$ is displayed in Fig. 4(a) for 14 applied voltages of up to $1000 \mathrm{~V}$ and an electric field up to $\sim 36 \mathrm{MV} / \mathrm{m}$ or $\sim 12 \%$ of the breakdown field strength. The curves all show an initial exponential rise in current before $0.2 \mathrm{~s}$, with a time constant $\tau_{Q} \approx(0.20 \pm 0.02) \mathrm{s}$, which is attributed to either the response time of the voltage supply [15] or to the details of the charge injection process [26]. Additional data taken at higher electric fields might be able to distinguish between the instrumentation and various injection behaviors [26]. This rapid rise is followed by an exponential decline with an average polarization decay time $\tau_{P}=(0.80 \pm 0.05) \mathrm{s}$, independent of the applied electric field up to $\sim 36 \mathrm{MV} / \mathrm{m}$. Such a rapid polarization decay time is consistent with the fact that polyethylene has a non-polar monomer.

The long-term electrode current data (see Fig. 4(b)) are modeled with a modified version of (B8). The fit (green curve) is the sum of a constant saturation current of $J_{\text {sat }} \sim 1.5 \cdot 10^{-14} \mathrm{~A}$ and an inverse power law term, $\left(J_{d}^{o} \cdot t^{-1}\right)$ with $\int_{d}^{o}=3 \cdot 10^{-11} \mathrm{~A}$, used to model the sum of $\sigma_{\text {diffusion }}$ and $\sigma_{\text {dispersive }}$ terms in (3a) as $\alpha \rightarrow 0$. Since the current is still decreasing after elapsed times up to $\sim 5$ days, we can conclude $\tau_{\text {transit }} \approx 3 \cdot 10^{5} \mathrm{~s}$. The data for times before $\sim 50 \mathrm{~s}$ in Fig. 4(b) are not fit well, because the polarization and injection timedependant terms were not included in this fit. The estimated fitting parameters for $\tau_{Q}, \tau_{P}, \tau_{\text {transit }}, \sigma_{\text {sat }}, \sigma_{\text {pol }}$, and $\sigma_{\text {diffusion }}$ plus
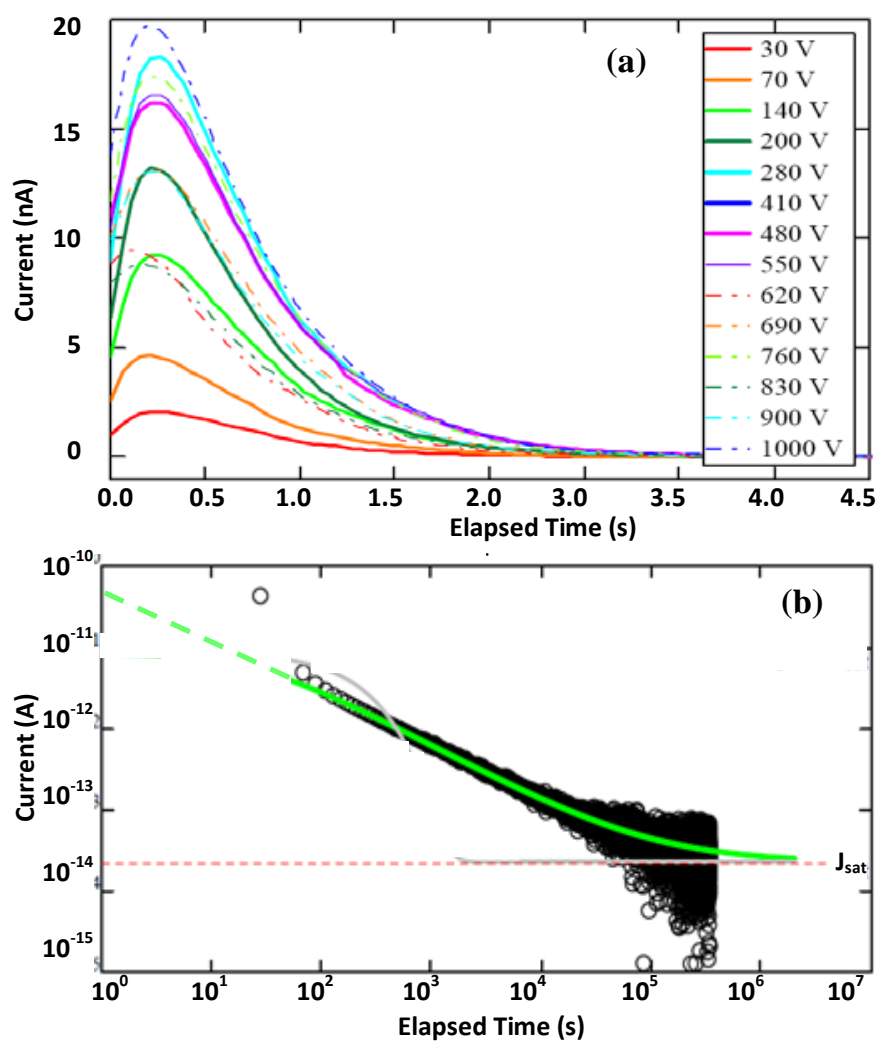

Fig. 4. Time dependence of the sample current under applied voltage for LDPE samples and $100 \mathrm{~V}$ applied voltage. (a) Initial current decay due to internal polarization for a series of 14 applied electric fields. Models are based on (5). (b) Rear electrode current data for times up to $\sim 5$ days. The data are shown in black. The model based on (3a) is shown in green. The maximum current and minimum current are shown as dotted lines for reference.

$\sigma_{\text {dispersive }}$ are in good agreement with previous measurements of LDPE [15,23,24].

\section{CONCLUSION}

The CVC has undergone modifications which improve the precision of conductivity measurements by nearly an order of magnitude. Uncertainties in measured values of current and conductivity are consistent with detailed error analysis of the system, reflecting the increased precision due to those modifications. Planned use of higher voltage stable battery supplies will lead to further increased precision of almost two orders of magnitude approaching $\sim 4 \cdot 10^{-23}(\Omega \text {-cm })^{-1}$; this precision is near fundamental limits set by thermal Johnson noise and RIC from natural background cosmic radiation. It is now clear that careful application of sufficient duration for both CVC and CSC methods can ultimately measure conductivities and decay times well beyond limits typically required for spacecraft charging applications of $\gtrsim 4 \cdot 10^{-22}(\Omega$ $\mathrm{cm})^{-1}$.

The time-dependant rear electrode current data are fit with a model that includes explicit contributions for the free charge carrier current (saturation and RIC currents), terms associated with the evolution of the spatial distribution of discrete charges trapped in localized states (diffusion, dispersion, transit currents), and displacement currents resulting from both transient response of bound charge (polarization and AC currents) and changes in the electric field from either applied 
electric fields or accumulated charge distributions. The measured values for LDPE acquired with this CVC system are fit well with this model and lead to fitting parameters consistent with values obtained in previous studies. Inclusion of displacement currents in the model-which have large initial magnitudes compared to the equilibrium free carrier and evolving charge distribution currents, but are relatively shortlived-provide an important explanation of why short-term fluctuations in the applied voltage can result in currents that dominate the CVC system noise.

In addition, the theoretical model clearly identifies fundamental differences between the CVC and CSC methods. Most important are: (i) the differences in the surface voltage due to differences in the type of charge injection and (ii) the inclusion of a finite saturation current for CVC measurements. It also allows determination of which current terms and injection voltages are relevant for either CVC or CSC methods.

In the final analysis, to determine whether CVC or CSC test methods are most appropriate for spacecraft charging applications requires a more detailed knowledge of the dynamics of the specific problem. Situations with uniform continuous charge injection are best studied with CVC measurements. For example, a continuous consistent charge particle flux from ambient space radiation may be better characterized by application of a constant voltage over long enough time scales to reach equilibrium saturation currents. By contrast, transient incident space fluxes due to environmental changes (e.g., solar flares, coronal mass ejections, or dynamic magnetic fields), geometry changes (e.g., spacecraft rotations, orbits or eclipses), or even material modification (e.g., contamination, oxidation, or radiation damage) may be better characterized by pulsed time-of-flight CSC test methods. That is to say, the choice of appropriate conductivity test methods and their duration is driven by comparisons to the relevant time scales of the specific space environment application and the material response.

\section{APPENDIX A: ERROR ANALYSIS FOR THE CVC SYSTEM}

The precision in conductivity measurements using (1) is determined from the random uncertainties in four measured quantities-A, $D, J_{\text {elec }}$ and $V_{a p p}$-as given by (2). The uncertainties for the CVC system associated with these four measurements are discussed below.

The $(1.59 \pm 0.03) \mathrm{cm}$ diameter oxygen-free, highconductivity (OFHC) $\mathrm{Cu}$ electrodes have an effective contact area of $(1.98 \pm 0.08) \mathrm{cm}^{2}$ with an accuracy of $\pm 4 \%$ [6]. The contact area of the electrode has been made more reproducible from run to run and sample to sample by the addition of a sample clamping fixture. To insure proper contact between the electrodes and the sample surface, a four spring clamping mechanism — as shown in Fig. 1(b)—was added to provide consistent and repeatable sample pressure [6], adjustable over the 140-700 $\mathrm{kPa}$ range recommended in ASTM D-257 [7]. Chotherm $^{\mathrm{TM}}$ insulation was also installed, to insure that the grounding plate remained electrically isolated, but in good thermal contact with the cryogen reservoir (see Fig. 1(b)). Precision for area $A$, as limited by variations in clamping; is estimated as $\sim 1 \%$.

Typical sample thicknesses of $10 \mu \mathrm{m}$ to $200 \mu \mathrm{m}$ were measured with a standard digital micrometer with a resolution of $\pm 0.3 \mu \mathrm{m}$, with relative errors of $0.1 \%$ to $3 \%$. Variations in thickness across typical samples were comparable to or larger than this measurement error.

To further improve the quality of the data, an adaptive smoothing algorithm was developed to process the measured current and voltage data. The time interval between acquisitions of sets of current (or voltage) data points was typically between $0.1 \mathrm{~s}$ and $10 \mathrm{~s}$, depending on how fast the current was changing. The algorithm intelligently adjusted the time window or bin width of data sets to average over, based on the rate at which the current (or voltage) was changing (refer to [6] for details).

The estimated precision for current measurements, $(\Delta I /|I|)$, is $\lesssim 0.1 \%$ at $>1 \cdot 10^{-11} \mathrm{~A}$ and $\gtrsim 20 \%$ at $\lesssim 1 \cdot 10^{-15} \mathrm{~A}$. This follows from an expression for the relative precision from the measured standard deviation of the mean current for a set of $N_{I}$ measurements (typically 1000), made using our electrometer (Keithley, Model 616) and data acquisition card (DAC) (National Instruments, Model 6221; 16-bit, $100 \mathrm{kHz}$ ) at a rate of $f_{I}$ (typically $5 \mathrm{kHz}$ ) over a sampling period $N_{I} / f_{I}$ (typically $0.2 \mathrm{~s}$ ) for a current range, $10^{R}$, of $10^{-6} \mathrm{~A}$ to $10^{-15} \mathrm{~A}$ with sensitivity setting $S$ :

$$
\begin{aligned}
\frac{\Delta I}{|I|}= & {\left[N_{\text {Bin }}\left(N_{I}-1\right) \cdot \operatorname{Min}\left(1, \frac{2}{T_{\text {Rise }} \cdot f_{I}}\right)\right]^{-1 / 2}\left\{\left[\frac{\Delta I_{\text {rel }}^{\text {elec }}}{|I|}+\frac{\Delta I_{\text {rel }}^{D A Q}}{|I|}\right]\right.} \\
& \left.+\left\{[1.4-0.4(3-S)] \frac{\Delta I_{o}^{\text {elec }}}{|I|}+\cdot 10^{(S-2)} \frac{\Delta I_{o}^{D A Q}}{|I|}\right\} 10^{R}\right\}, \quad(\mathrm{A} 1)
\end{aligned}
$$

in terms of absolute $\left(\Delta I_{o}\right)$ and relative $\left(\Delta I_{\text {rel }} /|I|\right)$ errors for the electrometer and DAC $[6,28]$. At typical measured low currents, the contributions to uncertainties due to the electrometer dominate those from the DAC [6]. The initial term in square brackets, in (A1), accounts for the reduction in the uncertainty of the mean by sampling the electrometer $N_{I}$ times for each current data set and $N_{B i n}$ data sets averaged in the binning/smoothing algorithm. The standard deviation of the mean of each current data set sampled is reduced by a complicated function proportional to $\left(N_{I}-1\right)^{-1 / 2}$ that depends on the number of data points sampled by the DAC, the sampling rate of the DAC $f_{I}$, and the electrometer rise time, $T_{\text {Rise }}$. The factor $\left(2 / T_{\text {Rise }} f_{I}\right)$ is the number of samples that can be measured for a given response time at the Nyquist limit for a given sampling rate. Since this factor cannot exceed unity, the Min function returns the minimum value of unity or $\left(2 / T_{\text {Rise }} f_{I}\right)$. This corrects for the limitation that, at lower range settings, the sampling time $1 / f_{I}$ is less than the response time of the electrometer and oversampling results.

The relative error in the measured standard deviation of the mean of the applied voltage is

$$
\frac{\Delta V}{|V|}=\left(N_{V}-1\right)^{-\frac{1}{2}} \cdot\left[\frac{\Delta V_{o}}{|V|}+\frac{\Delta V_{r e l}}{|V|}\right]
$$

A set of $N_{V}$ (typically 100) measurements of the voltage monitor are made at a rate $f_{V}$ (typically $1 \mathrm{kHz}$, which is assumed to be less than the inverse of the response time of the 
voltage supply monitoring circuit). The uncertainties in (A2) are a combination of uncertainties from the DAC and programmable voltage supplies. The relative voltage dependent term, $\Delta V_{\text {rel }} /|V|$, includes: the voltage supply stability, load regulation, and AC line regulation; the voltage supply circuit converting the programming voltage from the DAC to the high voltage output; and the voltage supply circuit converting the high voltage output to the voltage monitor signal passed to the DAC. The constant error term, $\Delta V_{o}$, includes: variations of \pm 1 least significant bit (LSB) in the 16 bit analog output signal of the DAC into the programming voltage of the power supply and from the DAC derived from the high voltage monitoring signal of the power supply; the DAC thermal error; the maximum ripple in the high voltage output of the voltage supply; and variations due to random thermal fluctuations in the voltage.

Three power supplies have been used in different CVC tests, and are considered in detail in [6]. Two programmable DC voltage sources were used: a high voltage supply (Acopian, Model P020HA1.5; $20 \mathrm{kV}$ at $1.5 \mathrm{~mA}$ ) with $\Delta V_{o}=4 \mathrm{~V}$ and $\Delta V_{\text {rel }} /|V|=0.7 \%$ and a medium voltage supply (Bertan, Model 230-01R; $1 \mathrm{kV}$ at $15 \mathrm{~mA}$ ) with $\Delta V_{o} \approx 250 \mathrm{mV}$ and $\Delta V_{\text {rel }} /|V| \approx 0.1 \%$. At voltages below $400 \mathrm{~V}$ using the programmable DC voltage sources, the instrumental precision depends primarily on the DAC, while above this voltage errors from the voltage supply increase to $\sim 2 \mathrm{X}$ the DAC error.

Uncertainties from the applied voltage were substantially reduced using a third custom voltage source. A very lownoise low-voltage battery source constructed of twelve nine volt Duracell Professional Alkaline batteries in series, produced an applied voltage of approximately $102.5 \mathrm{~V}$ with $\Delta V_{o} \approx 16 \mathrm{mV}$ and $\Delta V_{\text {rel }} /|V| \lesssim 0.02 \%$. (For a similar $1000 \mathrm{~V}$ battery supply being built, $\Delta V /|V| \lesssim 15$ ppm [17], Uncertainties result largely from the voltage monitoring circuit which include: variations in \pm 1 LSB in the 16 bit signal into the analog input of the DAC; the DAC thermal error; instabilities and drift of thin film metal resistors in the 1:100 voltage divider circuit (see Fig. 1(a)); and calibration of the voltage divider circuit with an accuracy of $\sim 0.01 \%$. Long time scale voltage variation shows a typical $(30 \pm 2) \mathrm{mV} / \mathrm{hr}$ decline due to battery discharge and a $0.01 \%$ deviation from the linearity, resulting largely from the uncertainties in the voltage monitoring and DAC. On a short time scale, the voltage data show a $4 \mathrm{mV}$ or $20 \mathrm{ppm}$ deviation from the linear fit to the decay. Variation in accuracy of the applied voltage (due primarily to long-term drift) are directly monitored with the DAC and compensated for in the conductivity calculations; therefore, they do not contribute to the precision of the conductivity.

\section{APPENDIX B: TIME-DEPENDANT CONDUCTIVITY}

Based on (1), determination of a time dependant conductivity using the CVC method follows from measurement of the current density measured at the rear electrode, $J_{\text {elec }}(t)$. This is a complicated function of time, comprised of several component currents dependant on different aspects of the dielectrics. From the AmpereMaxwell equation this rear electrode current includes two contributions, the free charge transport current density, $J_{\text {elec }}^{c}$, and the charge displacement current density, $J_{\text {elec }}^{\text {displacement }}$,

$$
\begin{aligned}
J_{\text {elec }}(t) & =J_{\text {elec }}^{c}(t)+J_{\text {elec }}^{\text {displacement }} \\
& =\sigma(t) F(t)+\left[\epsilon_{o} \frac{\partial \epsilon_{r}(t)}{\partial t} F(t)+\epsilon_{o} \epsilon_{r} \frac{\partial F(t)}{\partial t}\right]
\end{aligned}
$$

It is convenient to consider these various contributions in terms of time-dependant functions for conductivity $\sigma(t)$, relative dielectric permittivity $\epsilon_{r}(t)$, and electric field $F(t)$. The general functional form and physical origins of these time-dependant terms, as related to the CVC method, are discussed in [26]; also see [6], [19], [21] and [23]. Numerous theoretical models for CVC currents, based on dynamic bulk charge transport equations developed for electron and hole charge carriers have been advanced to predict the time, temperature, dose, dose rate, and electric field dependence of the electrode current and surface voltage [22,26,32,35]. The most promising theories for explaining electrical behavior in insulating polymers are based on hopping conductivity models developed to understand charge transport in disordered semiconductors and amorphous solids [32,36]. These theories assume that electrons or holes are the primary charge carriers and that their motion through the material is governed by the availability of localized states treated as potential wells or traps in a lattice. These models make direct ties to the interactions between injected charge carriers-which are trapped in localized states in the HDIM-and the magnitude and energy dependence of the density of those localized trap states within the band gap; to the carrier mobility; and to the carrier trapping and de-trapping rates. Overviews of the models are provided by Molinié [35,36] and Sim [26]; more detailed discussions are presented by Sim [23], Wintle [32] and Kao [37].

We begin by considering the first term in (B1), which models how easily an excess free charge injected into the material from the electrode can move through the material in response to an electric field and is proportional to a timedependant particle current conductivity, $J_{\text {elec }}^{c}(t)=\sigma(t) F(t)$. A general form of conductivity in HDIM, with explicit time dependence, takes the form

$\sigma(\mathrm{t})=\left[\begin{array}{c}\sigma_{\text {Sat }}+\sigma_{\mathrm{RIC}}(\mathrm{t})+\sigma_{\mathrm{AC}}(v)+\sigma_{\text {pol }}^{\mathrm{o}} \mathrm{e}^{-\mathrm{t} / \tau_{\mathrm{pol}}}+\sigma_{\text {diffusion }}^{\mathrm{o}} \mathrm{t}^{-1}+ \\ \sigma_{\text {dispersive }}^{\mathrm{o}} \mathrm{t}^{-(1-\alpha)} \Theta\left(\tau_{\text {transit }}-\mathrm{t}\right)+\sigma_{\text {transit }}^{\mathrm{o}} \mathrm{t}^{-(1+\alpha)} \Theta\left(t-\tau_{\text {transit }}\right)\end{array}\right]$

as discussed in [26] and [32] and detailed in [23] and extensive references therein. $\Theta(x)$ is the Heaviside step function.

We provide a brief summary of each contribution to (B1), with emphasis on their relation to the CVC and CSC methods. The conductivity terms are:

Saturation Conductivity: The saturation conductivity, $\sigma_{S a t} \equiv q_{e} n_{e} \mu_{e}$, results from the very long time scale equilibrium conductivity without radiation induced contributions, sometimes referred to as drift conduction. This represents the steady state drift of free charge across the bulk insulator, driven by an applied field. For this term, the equilibrium free carrier density, $n_{e}$, and the free electron mobility, $\mu_{e}$, are independent of time and position. In practice the saturation current is less than an upper bound set by the dark current conductivity for materials with no internal space charge, since 
this internal space charge can inhibit the transport of charge carriers across the material $[23,26]$. Stated another way, the dark current conductivity results when the trap states are fully filled, whereas the saturation current depends only on the fraction of filled trap states for a given experimental configuration.

Note that $\sigma_{\text {Sat }}(t \rightarrow \infty) \rightarrow 0$ once injection ceases (as is the case for the CSC method), but asymptotically approaches a constant value when there is continuous charge injection (as is the case for the CVC method).

Radiation Induced Conductivity: Another steady-state conduction mechanism, called photoconductivity or radiation induced conductivity (RIC), involves excitation of charge carriers by external influences-including electron, ion and photon high energy radiation-from either extended or localized states into extended states. The Rose [38], Fowler [39], and Vaisberg [40] theory provides a good model of RIC, as discussed in the context of the spacecraft charging materials characterization in [23], [26] and [30].

During electron beam deposition for the CSC method, RIC is active only in the RIC region encompassing material from the injection surface up to the penetration depth of the electron beam, $R\left(E_{i n j}\right)$, but diminishes quickly after the beam is turned off. We neglect the time dependence of RIC times soon after the beam is turned on or off. RIC is not active for the CVC method, where charge is injected via an electrode rather than an incident charge beam; RIC does enter the discussion for CVC measurements here as an effective noise term from cosmic background radiation.

Transient Conductivity: Next we consider three transient conductivity terms — diffusion, dispersion and transit—all due to the redistribution of the injected charge distribution trapped in the material. In HDIM, the concept of "free" versus "bound" charge is rather ambiguous, since injected charge can be transported across the material on very long time scales but can also reside in trap states for long periods of time during transit. On short time scales, these conductivity terms are more properly consider as displacement currents resulting from the change in the internal electric field from the trapped charge due to the motion of quasi-free trapped space charge distributions within the material. However, for clarity of presentation, we group them here with the "free" charge transport terms.

Space charge effects can be significant as traps are filled with injected charge and can inhibit further motion of the carriers. This leads to a fundamentally different behavior for the diffusion term for CSC and CVC methods. For CSC methods, the time required to inject the charge is usually much shorter than the conductivity measurement or transit times, so the pulsed injection leads to a localized (in both time and depth) injected charge distribution that propagates across the sample under the influence of the electric field; the CSC method falls into a "time-of-flight" category. In the long time limit for CSC, the injected charge is cleared from the sample. By contrast, the CVC method produces a continuous charge injection and ultimately a finite, uniform equilibrium charge distribution across the sample proportional to the applied voltage.
Diffusive Conductivity: Diffusive conductivity results from the advance of the charge front or the centroid of the trapped space charge distribution via diffusion or hopping of trapped carriers. This transient conduction mechanism is driven by spatial gradients in the charge distribution. For HDIM, the space charge is in trap states most of the time (i.e., the retention time(s) is greater than the trap filling time(s)), so the conduction mechanisms relevant to this process are largely governed by transitions to and from trap states; that is, diffusion in HDIM proceeds by thermally assisted hopping $[32,41,42]$ or variable range hopping [43-45] mechanisms. For one-dimensional motion in HDIM, trapped state diffusion is inversely proportional to $t, \sigma_{\text {diffusion }}(t) \equiv \sigma_{\text {diffusion }}^{o} \cdot t^{-1}$. For time-independent charge injection, once the centroid of the trapped charge distribution reaches the rear electrode, at times $\gtrsim \tau_{\text {transit, }}$, the diffusive conductivity no longer contributes to $\sigma(t)$. This is the case for both CVC (constant injection at long times) and CSC (no injection after short times) methods.

Dispersive and Transit Conductivity: $\sigma_{\text {dispersive }}(t) \equiv$ $\sigma_{\text {dispersive }}^{o} \cdot t^{-(1-\alpha)}$ (for $t<\tau_{\text {transit }}$ ) and $\sigma_{\text {transit }}(t) \equiv \sigma_{\text {transit }}^{o} \cdot t^{-(1+\alpha)}$ (for $t>\tau_{\text {transit }}$ ) are two parts of a contribution to conductivity that results from the broadening of the spatial distribution of the space charge participating in transport through a coupling with the energy distribution of trap states. For HDIM, charge transport of trapped space charge progresses by hopping mechanisms involving localized trap states (e.g., thermally assisted or variable range hopping). These mechanisms lead to a power law time-dependence, characterized by the dimensionless dispersion parameter, $\alpha$, related to the trap filling and release rates, which is a measure of the width of the trap state energy distribution [26,32,46,47]. Note, when $\alpha \rightarrow 0$ for dispersion less materials, diffusive, dispersive and transit conductivities all have $t^{-1}$ dependence and cannot be easily distinguished [32,37]. For dispersive and transit contributions, the space charge distribution broadens with time, progressing towards a uniform distribution of space charge across the dielectric. The transition from dispersive to transit behavior, and the concomitant drop in the displacement current, occurs at a time $\tau_{\text {transit }}$ at which the first of the injected charge carriers have traversed the sample, thereby reducing the magnitude of the charge distribution that can further disperse $[46,48]$. The exact nature of the broadening is different for the pulsed and stepped charge distributions that occur for CVC and CSC methods.

Polarization Conductivity: Next we consider the result of the time-dependant permittivity in the second term of (B1), expressed as an effective conductivity proportional to the electric field. In dielectric materials, a displacement conduction mechanism results from the time-dependant response of the material as the internal bound charge of the dielectric material rearranges in response to an applied electric field on a time scale $\tau_{\text {pol }}[24,26]$. No net charge is transferred across the material; rather the transient polarization current results primarily from the reorientation of molecular dipoles and the movement of ionic charge from one part of the sample to another in response to the applied field. In a simple relaxation time model of this charge displacement, the current 
in a parallel plate geometry for a constant applied voltage can be expressed as a time-dependant effective polarization conductivity [24],

$$
\sigma_{p o l}(t)=\sigma_{p o l}^{o} \cdot e^{-t / \tau_{p o l}}
$$

AC-loss conductivity: The polarization current is essentially a very low frequency AC-loss conductivity term. Higher frequency terms result from higher frequency periodic applied voltages and are not directly applicable for the CVC or CSC methods. $\sigma_{A C}(v)$ is a frequency-dependant AC conduction that is a measure of the dielectric response to a periodic applied electric field, and is only active for periodic charge injection [32].

Low frequency terms, such as produced by a small sinusoidal ripple from an applied voltage sources, can be treated as a time varying applied field $\left(V_{\text {ripple }} / D\right) \cdot \cos (\omega t)$ in (B6) for the displacement current discussed below, with a constant low frequency permittivity, $\epsilon_{o} \epsilon_{r}$. For a low frequency ripple with frequency $\omega \ll \tau_{Q}^{-1}$, the resulting displacement current from the last term of (B1) is

$$
J_{\text {ripple }}^{\text {displacement }}(t)=\epsilon_{o} \epsilon_{r} \frac{\partial F(t)}{\partial t}=\frac{\epsilon_{o} \epsilon_{r} \omega}{D} V_{\text {ripple }} \sin (\omega t)
$$

This leads to an additional error in conductivity of

$\frac{\Delta \sigma_{\text {Ripple }}}{|\sigma|} \approx \frac{J_{\text {ripple }}^{\text {displament }}}{J_{\text {elec }}^{c}} \approx\left(\omega \tau_{\mathrm{Q}}\right)^{-1} \frac{V_{\text {ripple }}}{V_{\text {app }}}$

For a typical value of $\tau_{Q}=0.2 \mathrm{~s}$ (see Section V.B) and a $60 \mathrm{~Hz}$ ripple, the relative error from this conductivity term is $\sim 10 \%$ of $\Delta V_{\text {rel }} /|V|$, in very good agreement with the reduce error observed in Fig. 3 as ripple is reduced.

Displacement Current: The final term to consider in (B1) is the displacement current proportional to the time derivative of the electric field $F$, where $F$ is given by the sum of the applied field and the self-induced field due to the interaction of accumulated charge and its image charge on the rear electrode. Calculation of the displacement current requires an expression for the time-dependant field, $F(t)$. A particularly simple model for the surface field as a function of elapsed time follows a charging capacitor model,

$\mathrm{F}(t)=F_{a p p}\left(1-e^{-t / \tau_{Q}}\right)$,

for simple charge accumulation on the surface, with an associated displacement current from (B1), of

$J_{\text {displacement }}(t)=\left(\frac{-\varepsilon_{0} \varepsilon_{\mathrm{r}}}{\tau_{Q}}\right) \mathrm{F}_{\mathrm{app}} e^{-t / \tau_{Q}}=\left(\frac{-\tau_{\text {transit }}}{\tau_{Q}}\right) J_{b} e^{-t / \tau_{Q}}$.

Here $\tau_{Q}$ is an injection time constant characterizing the injection current density, $J_{i n j}(t)$, which is not to be confused with the decay or transit time constant, $\tau_{\text {transit. }}$ Physically $\tau_{Q}$ can model either the rise time of the applied voltage power supply or a time- (or surface voltage-) dependent injection current density for charge injected into the upper surface.
A more general treatment of the long-term displacement currents has been developed by Walden [49] and Wintle [50], who consider a general form for the electrode injection current density as a function of applied electric field. They consider a very general expression for the injection voltage, which includes the simple exponential model used here, as well as more sophisticated models for space charge limited conduction, Poole-Frenkel conduction for Schottky or thermionic emission, Fowler-Nordhiem injection for tunneling type emission, and other models.

A similar model for electron beam charge injection suitable for CSC methods has been developed in $[19,21,26]$ using the Walden and Wintle formalism. This produces a similar result with $\tau_{Q}$ interpreted as a characteristic time to acquire sufficient surface charge for the electron yield to approach unity [26]. Different expressions have been found for positive charging with electron yield greater than unity and for negative charging with electron yield less than unity [20].

For the longer-term time-independent conductivity estimated above and for general voltage expressions for the parallel plate geometry, it has been shown that this general displacement current has the form

$J_{\text {elec }}(t)=J_{\text {inj }}\left\{1+t\left[\tau_{W}+t\right]^{-1}\right\}$,

where $\tau_{W}$ is a generalized decay time found as a function of the time dependence of the electric field $[26,49,50]$. (B6) has obvious similarities to (B2) when $\sigma_{R I C}, \sigma_{A C}$ and $\sigma_{p o l}$ contributions are neglected and $\alpha \rightarrow 1$. This has been reviewed in considerable detail in [26] and [23]. Wintle [32], Kao [37], and Sim [23] and others derive similar expressions for the rear electrode current based on general rate equation models.

Recall, there are additional displacements currents related to the changes in the internal electric field as the distribution of quasi-free trapped space charge within an HDIM evolves; these include the diffusion, dispersion and transit conductivities discussed above, and have already been included in the expressions for time-dependent conductivity, (B2).

\section{ACKNOWLEDGMENT}

This work was inspired by collaborations with $\mathrm{R}$. Frederickson and is dedicated to his legacy. We gratefully acknowledge work on the CVC chamber and data acquisition by S. Hart and R. Hoffmann and on the data analysis algorithm by J. Hodges.

\section{REFERENCES}

[1] D. Hastings, and H. Garrett, Spacecraft-environment Interactions, Cambridge University Press, 1996.

[2] D. Ferguson, "New Frontiers in Spacecraft Charging," IEEE Trans. Plasma Sci., vol. 40, no. 2, pp. 1-5, 2012.

[3] A.R. Frederickson and J.R. Dennison, "Measurement of Conductivity and Charge Storage in Insulators Related to Spacecraft Charging," IEEE Trans. on Nuclear Sci., Vol. 50, No. 6, pp. 2284-2291, 2003.

[4] NASA Technical Handbook, "Mitigating In-Space Charging Effects-A Guideline,” NASA-STD-4002A, 2011.

[5] C.K. Purvis, H.B. Garrett, A.C. Whittlesey, and N.J. Stevens, "Design Guidelines for Assessing and Controlling Spacecraft Charging Effects," NASA Tech. Paper 2361, 1984.

[6] J. Dekany, A. M. Sim, J. Brunson, and J.R. Dennison, "Electron Transport Models and Precision Measurements in a Constant Voltage 
Chamber," Proc. $12^{\text {th }}$ Spacecraft Charging Techn. Conf., (Kitakyushu, Japan, May, 2012).

[7] ASTM D 257-99, "Standard Test Methods for DC Resistance or Conductance of Insulating Materials" (Am. Soc. for Testing and Materials, West Conshohocken, PA 19428, 1999).

[8] A.R. Frederickson, C.E. Benson, and J.F. Bockman, "Measurement of Charge Storage and Leakage in Polyimides," Nuclear Instruments and Methods in Physics Research Section B: Beam Interactions with Materials and Atoms, vol. 208, no. 7, pp. 454-460, Aug. 2003.

[9] N.W. Green, A.R. Frederickson and J.R. Dennison, "Charge Storage Measurements of Resistivity for Dielectric Samples from the CRRES Internal Discharge Monitor," IEEE Trans. Plasma Sci., 34(5), 19731978, 2006,

[10] A.R. Frederickson and J.R. Dennison, "Measurement of Conductivity and Charge Storage in Insulators Related to Spacecraft Charging," IEEE Trans. on Nuclear Sci., vol. 50, no. 6, 2284-2291, 2003.

[11] A.R. Frederickson and C.E. Benson, "Improved Testing Procedures For Spacecraft Discharge", Proc. $7^{\text {th }}$ Spacecraft Charging Tech, Conf. (ESTEC, Noordwijk, The Netherlands, 2001).

[12] J.R. Dennison, P. Swaminathan, R. Jost, and J. Brunson, N.W. Green, and A.R. Frederickson, "Proposed Modifications To Engineering Design Guidelines Related To Resistivity Measurements And Spacecraft Charging," Proc. $9^{\text {th }}$ Spacecraft Charging Tech. Conf., (Epochal Tsukuba, Tsukuba, Japan, 2005).

[13] P. Swaminathan, A.R. Frederickson, J.R. Dennison, A.M. Sim, J. Brunson and E. Crapo, "Comparison of Classical and Charge Storage Methods for Determining Conductivity of Thin Film Insulators,” Proc. 8th Spacecraft Charging Tech. Conf., NASA Marshall Space Flight Center, Huntsville, Alabama, 2003.

[14] ASTM D 618, "Standard Practice for Conditioning Plastics for Testing" (Am. Soc. Testing and Materials, West Conshohocken, PA 19428, 2001).

[15] J. Brunson, "Measurement of Charge Decay Time and Resistivity of Spacecraft Insulators Using Charge Storage Method and Application to Theoretical Modeling of Charging Behavior of Insulators," $\mathrm{PhD}$ Dissertation; Physics Department; Utah State University, 2009.

[16] J. Dekany, "Evaluation of Constant Voltage Chamber Modifications," Senior Thesis, Physics Dept., Utah State Univ., Logan, UT, 2009.

[17] P. Lundgreen, J. Dekany, and J.R. Dennison, "Time Dependant Conductivity of Low Density Polyethylene," Am. Phys. Soc. Four Corners Sec. Meet., (Denver, CO, 2013).

[18] P. Swaminathan, "Measurement of Charge Storage Decay Time and Resistivity of Spacecraft Insulators," MS Thesis, Utah State Univ., Logan, UT, 2004.

[19] J.L. Hodges, "In Situ Measurements of Electron Beam Induced Surface Voltage of Highly Resistive Materials,” MS Thesis, Physics Dept.; Utah State Univ., 2012.

[20] G. Wilson, JR Dennison, A. Evans and J. Dekany "Electron Energy Dependent Charging Effects of Multilayered Dielectric Materials" IEEE Trans. Plasma Sci., to be published.

[21] J. L. Hodges, A. M. Sim, J. Dekany, G. Wilson, A. Evans, and JR Dennison "In Situ Surface Voltage Measurements of Layered Dielectrics," IEEE Trans. Plasma Sci., to be published.

[22] D. Min, S. Li, M. Cho, and A. R. Khan, "Investigation ioto Surface Potential Decay of Polyimide by Unipolar Charge Transport Model," IEEE Trans. Plasma Sci., to be published.

[23] A.M. Sim, "Unified Model of Charge Transport in Insulating Polymeric Materials,” PhD Thesis, Physics Dept., Utah State Univ., Logan, UT, 2013.

[24] J.R. Dennison and J. Brunson, "Temperature and Electric Field Dependence of Conduction in Low-Density Polyethylene,” IEEE Trans. Plasma Sci., 36(5), 2246-2252, 2008.

[25] J. Dekany, A. M. Sim, J. Brunson, and J. R. Dennison, "Electron Transport Models and Precision Measurements in a Constant Voltage Chamber," IEEE Trans. Plasma Sci., to be published.

[26] A.M. Sim and J. R. Dennison, "Comprehensive Theoretical Framework for Modeling Diverse Electron Transport Experiments in Parallel Plate Geometries," Paper Number, AIAA-2013-2827, $5^{\text {th }}$ AIAA Atmospheric and Space Environ. Conf., (San Diego, CA, June 24-27, 2013).

[27] Low Level Measurement Handbook, 6th Edition (Keithley Instruments, Inc., Cleveland, OH, 44139, 2004).

[28] Keithley Electrometer Model 616 Manual (Keithely Instruments, Inc., Cleveland, OH, 44139, 1983).

[29] C. Amsler, et al, "Review of Particle Physics," Phys. Lett. B, vol. 667, 1, 2008.
[30] J.R. Dennison, J. Gillespie, J.L. Hodges, R.C. Hoffmann, .J. Abbott, A.W. Hunt and R. Spalding, "Radiation Induced Conductivity of Highly-Insulating Spacecraft Materials," in Application of Accelerators in Research and Industry, Am. Instit. Phys. Conf. Proc. Series, Vol. 1099, ed. F.D. McDaniel and B. L. Doyle, (Am. Instit. of Phys., Melveille, NY, 2009), pp. 203-208.

[31] "Material Information--Polyethylene Low Density LDPE," .Goodfellow, Devon, PA, January 20, 2006.

[32] H.J. Wintle, "Conduction Processes in Polymers," in Engineering Dielectrics - Volume IIA: Electrical Properties of Solid Insulating Materials: Molecular Structure and Electrical Behavior, R. Bartnikas, Eds, (Am. Soc. Testing and Materials, Philadelphia, PA 19103, 1983).

[33] ASTM F495-99a, "Standard Test Method for Weight Loss of Gasket Materials Upon Exposure to Elevated Temperatures," (Am. Soc. Testing and Materials, West Conshohocken, PA 19428, 2011).

[34] ASTM D3755-97, "Standard Test Method for Dielectric Breakdown Voltage and Dielectric Strength of Solid Electrical Insulating Materials Under Direct-Voltage Stress," (Am. Soc. Testing and Materials, West Conshohocken, PA 19428, 2004).

[35] P. Molinié, "A Review of Mechanisms and Models Accounting for Surface Potential Decay,” IEEE Trans. Plasma Sci., 40(2), 167-176, 2012.

[36] P. Molinié, R. Hanna, T. Paulmier, M. Belhaj, B. Dirassen, D. Payan, and N. Balcon, "Photoconduction and radiation-induced conductivity on insulators: a short review and some experimental results," IEEE Trans. Plasma Sci., to be published.

[37] K.C. Kao, Dielectric Phenomena in Solids (Elsevier Academic, San Diego, 2004), pp. 41-114.

[38] A. Rose, 1951, "An Outline of Some Photoconductive Processes," RCA Review 12, 362.

[39] J. Fowler, 1956, "X-Ray Induced Conductivity in Insulating Materials," Proc. Royal Soc. London. Series A, Math. Phys. Sci. (1934-1990) 236, 464.

[40] S. E. Vaisberg, in Radiation Chemistry of Polymers, Ed. V. A. Kargin (Nauka, Moscow, 1973).

[41] A. Miller and E. Abrahams, "Impurity Conduction at Low Concentrations," Phys. Rev. 120, 745, 1960.

[42] V. Adamec and J.H. Calderwood, "On the determination of electrical conductivity in polyethylene,” J. Phys. D: Appl. Phys., 14, 1487-1494, 1981.

[43] N.F. Mott, "Conduction in Non-Crystalline Materials," Phil. Mag. 19, 835, 1969.

[44] N.F. Mott and E.A. Davis, Electronic Processes in Non-Crystalline Materials, $2^{\text {nd }}$ Ed. (Oxford Univ. Press, Oxford, 1979).

[45] N. Apsley and P.H. Hughes, "Temperature- and field-dependence of hopping conduction in disordered systems, II," Phil. Mag. 31 13271339, 1975.

[46] H. Scher and E. Montroll, "Anomalous Transit-Time Dispersion in Amorphous Solids," Phys. Rev. B 12, 2455, 1975.

[47] D. Monroe, "Band-Edge Condction in Amorphous Semiconductors," Disordered Semiconductors, 705, 1987.

[48] R. Zallen, The Physics of Amorphous Solids (Wiley, New York, NY) 1983.

[49] R.H. Walden, "A Method for the Determination of High-Field Conduction Laws in Insulating Films in the Presence of Charge Trapping," J. Appl. Phys. 43, 1178, 1972.

[50] H.J. Wintle, "Absorption Current, Dielectric Constant, and Dielectric Loss by the Tunneling Mechanism," J. Appl. Phys. 44, 2514,1973.

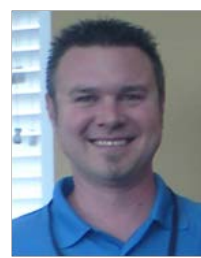

Justin Dekany is currently a graduate student at Utah State University in Logan, UT pursing an MS in physics. He received a BS degree in physics from USU in 2010. He has worked with the Materials Physics Group for four years on electron transport measurements, electrostatic discharge tests, electron emission measurements, and luminescence studies related to spacecraft charging. He has been the Lab Manager for the Materials Physics Group for the last two years. 
12

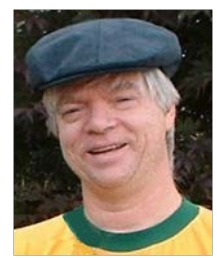

J. R. Dennison received the B.S. degree in physics from Appalachian State University, Boone, NC, in 1980, and the M.S. and Ph.D. degrees in physics from Virginia Tech, Blacksburg, in 1983 and 1985, respectively. He was a Research Associate with the University of MissouriColumbia before moving to Utah State University (USU), Logan, in 1988. He is currently a Professor of physics at USU, where he leads the Materials Physics Group. He has worked in the area of electron scattering for his entire career and has focused on the electron emission and resistivity of materials related to spacecraft charging for the last two decades.

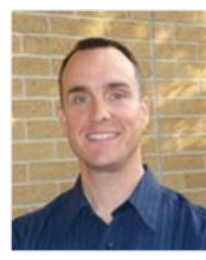

Alec Sim is a graduate student at Utah State University in Logan, UT pursing a $\mathrm{PhD}$ in physics. He received BS degree in physics from University of California-San Bernardino, CA in 2004 and an MS in physics from University of Kentucky, Lexington, KY in 2008. He has worked with the Materials Physics Group for six years on electron emission measurements and theoretical studies of electron transport in highly disordered insulating materials. He is currently an Assistant Professor in the Department of Physical Sciences at Irvine Valley College, Irving CA.

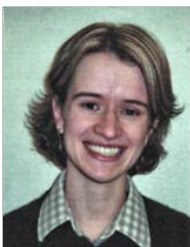

Jerilyn Brunson received her BS in 2003 and her PhD in 2008 in Physics from Utah State University in Logan, UT. She worked in the Materials Research group for six years, specializing in studies of the resistivity of highly insulating polymeric materials. She is currently a Research Physicist at the Naval Surface Warfare Center Dahlgren Division in Dahlgren, VA. 